\title{
A Practical and Scalable Method for Manufacturing JAK Inhibitor ASP3627
}

\author{
Shun Hirasawa, ${ }^{\dagger} *$ Takashi Kikuchi, ${ }^{\dagger}$ and Souichirou Kawazoe ${ }^{\dagger}$ \\ Process Chemistry Labs., Pharmaceutical Technology, Astellas Pharma Inc.,
}

160-2 Akahama, Takahagi-shi, Ibaraki 318-0001, Japan

\section{Present Addresses}

$\dagger$ Pharmaceutical Science \& Technology Labs., Pharmaceutical Technology, Astellas Pharma Inc.,

21 Miyukigaoka, Tsukuba-shi, Ibaraki 305-8585, Japan

\section{Supporting Information}

1. HPLC chromatograms of the $\mathrm{S}_{\mathrm{N}} \mathrm{Ar}$ reaction S2

2. ${ }^{1} \mathrm{H}$ and ${ }^{13} \mathrm{C}$ NMR spectra S4 


\section{HPLC chromatograms of the $S_{N} A r$ reaction}

Figure S1. The reaction mixture

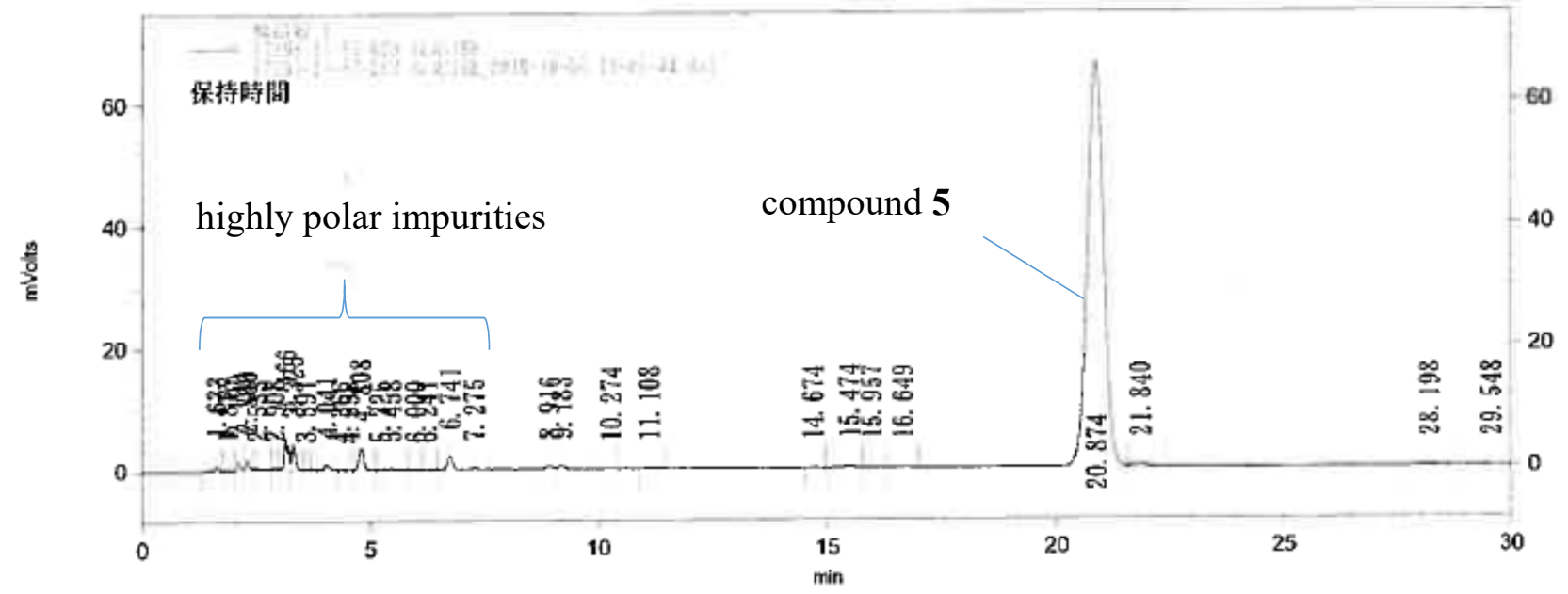

检出器 1 絬果

\begin{tabular}{|c|c|c|c|c|c|c|}
\hline Name & $\mathrm{Pk} \#$ & Retention Time & Area & Height & Intes, Codes & Area $\%$ \\
\hline & 1 & 1.63 & 6728 & 934 & BV & 0.357 \\
\hline & 2 & 1.87 & 2717 & 328 & VV & 0.144 \\
\hline & 3 & 2.10 & 9138 & 1358 & VV & 0.484 \\
\hline & 4 & 2.30 & 10170 & 1749 & VV & 0.539 \\
\hline & 5 & 2.53 & 4423 & 290 & VV & 0.234 \\
\hline & 6 & 2.91 & 2376 & 332 & vV & 0.126 \\
\hline & 7 & 3.17 & 30075 & 5266 & VV & 1.594 \\
\hline & 8 & 3.32 & 25398 & 4215 & VV & 1.346 \\
\hline & 9 & 3.59 & 666 & 117 & VB & 0.035 \\
\hline & 10 & 4.04 & 5995 & 825 & BB & 0.318 \\
\hline & 11 & 4.37 & 179 & 28 & BV & 0.009 \\
\hline & 12 & 4.56 & 256 & 41 & VV & 0.014 \\
\hline & 13 & 4.81 & 24197 & 3617 & vV & 1.282 \\
\hline & 14 & 5.22 & 535 & 71 & VV & 0.028 \\
\hline & 15 & 5.46 & 1990 & 197 & vV & 0.105 \\
\hline & 16 & 6.00 & 449 & 48 & VV & 0.024 \\
\hline & 17 & 6.24 & 659 & 72 & VB & 0.035 \\
\hline & 18 & 6.74 & 18291 & 2230 & $\mathrm{BV}$ & 0.969 \\
\hline & 19 & 7.27 & 3479 & 320 & VB & 0.184 \\
\hline & 20 & 8.92 & 5911 & 546 & $\mathrm{BV}$ & 0.313 \\
\hline & 21 & 9.18 & 6704 & 555 & VB & 0.355 \\
\hline & 22 & 10.27 & 270 & 29 & $\mathrm{BB}$ & 0.014 \\
\hline & 23 & 11.11 & 1834 & 134 & $\mathrm{BB}$ & 0.097 \\
\hline & 24 & 14.67 & 543 & 40 & $\mathrm{BB}$ & 0.029 \\
\hline & 25 & 15.47 & 6042 & 300 & BV & 0.320 \\
\hline & 26 & 15.96 & 2389 & 131 & VV & 0.127 \\
\hline & 27 & 16.65 & 1092 & 60 & VB & 0.058 \\
\hline & 28 & 20.87 & 1696684 & 66190 & $\mathrm{BV}$ & 89.927 \\
\hline & 29 & 21.84 & 9279 & 348 & VB & 0.492 \\
\hline & 30 & 28.20 & 4350 & 145 & $B B$ & 0.231 \\
\hline & 31 & 29.55 & 3922 & 123 & $\mathrm{BE}$ & 0.208 \\
\hline $\bar{A}^{\circ} \dot{A U}$ & & & 1886741 & 90639 & & 100.000 \\
\hline
\end{tabular}


Figure S2. Compound 5 after crystallization

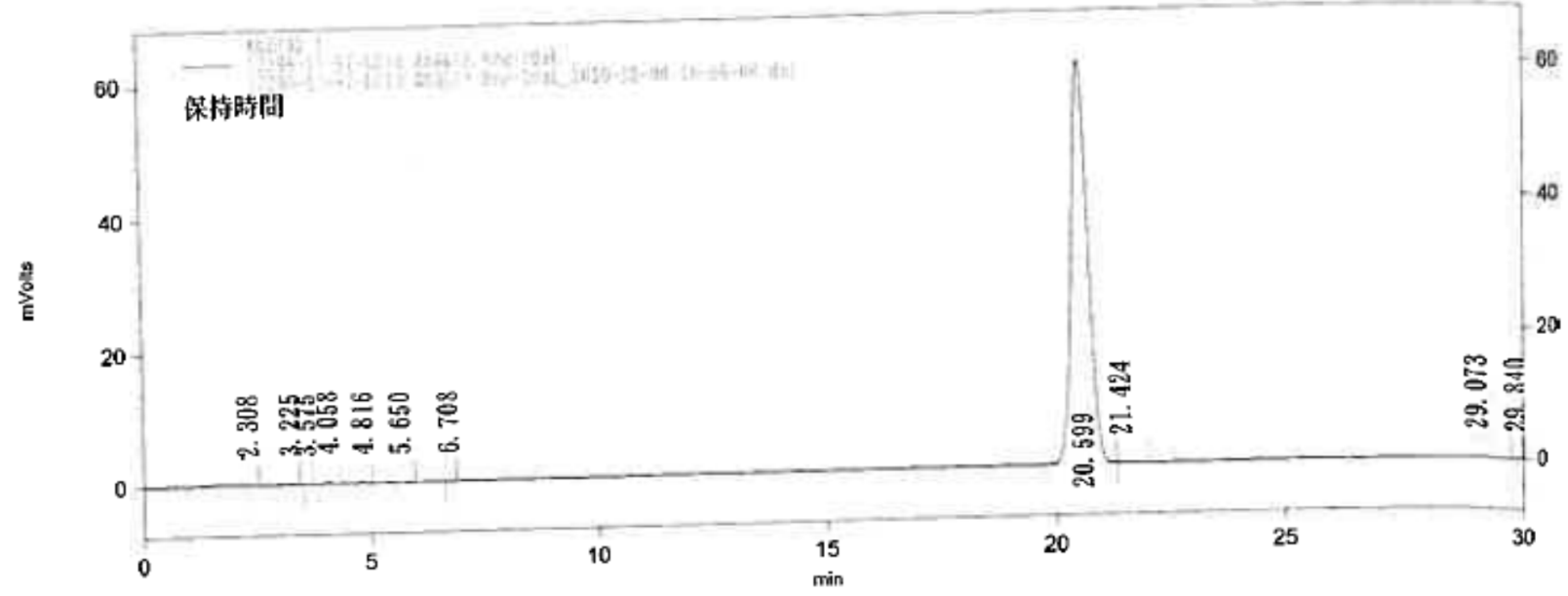

\begin{tabular}{|c|c|c|c|c|c|c|}
\hline $\begin{array}{l}\text { 㭘出器 } 1 \text { 結果 } \\
\text { Name }\end{array}$ & $\mathrm{Pk} \#$ & Retention Time & Area & Height & Integ. Codes & Area $\%$ \\
\hline & 1 & 2.31 & 111 & 23 & BB & 0.007 \\
\hline & 2 & 3.22 & 846 & 155 & $\mathrm{BB}$ & 0.055 \\
\hline & 3 & 3.57 & 199 & 44 & $\mathrm{BB}$ & 0.013 \\
\hline & 4 & 4.06 & 2397 & 396 & BB & 0.156 \\
\hline & 5 & 4.82 & 1132 & 181 & $\mathrm{BB}$ & 0.073 \\
\hline & 6 & 5.65 & 675 & 75 & $\mathrm{BB}$ & 0.044 \\
\hline & 7 & 6.71 & 175 & 26 & BB & 0.011 \\
\hline & 8 & 20.60 & 1529724 & 60580 & BV & 99.274 \\
\hline & 9 & 21.42 & 2767 & 134 & VB & 0.180 \\
\hline & 10 & 29.07 & 2878 & 100 & $\mathrm{BB}$ & 0.187 \\
\hline & 11 & 29.84 & 6 & 1 & $\mathrm{BE}$ & 0.000 \\
\hline $\bar{A}^{\circ} \mathrm{AU}$ & & & 1540910 & 61715 & & 100,000 \\
\hline
\end{tabular}


2. ${ }^{1} \mathrm{H}$ and ${ }^{13} \mathrm{C}$ NMR spectra

Compound 3

${ }^{1} \mathrm{H}$ NMR (DMSO- $d_{6}, 500 \mathrm{MHz}$ )

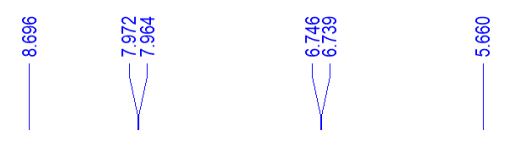

ํํำ

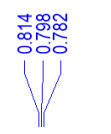<smiles>C[Si](C)(C)CCOCn1ccc2c(Cl)c(C#N)cnc21</smiles>
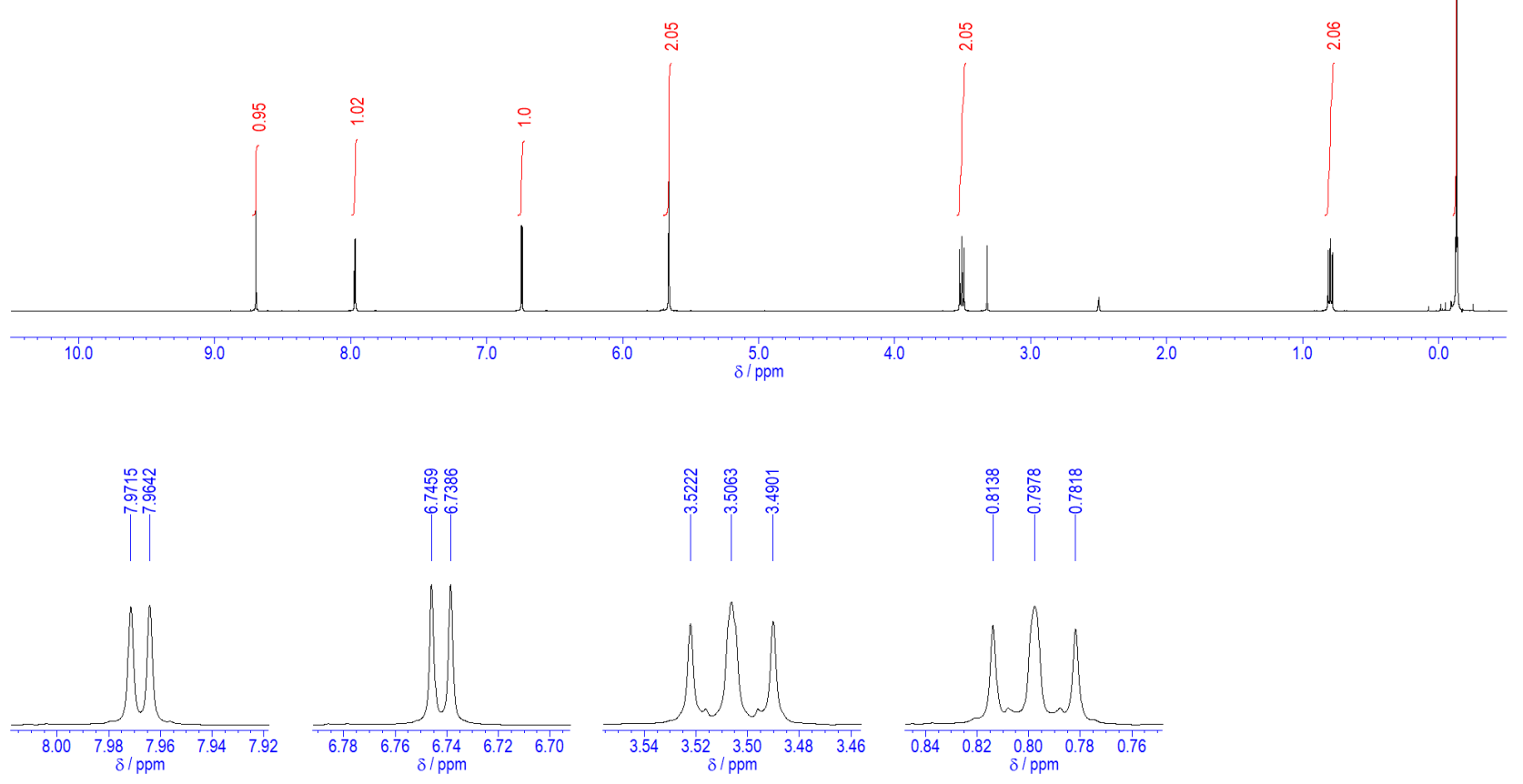


\section{Compound 3}

${ }^{13} \mathrm{C}$ NMR (DMSO- $d_{6}, 125 \mathrm{MHz}$ )

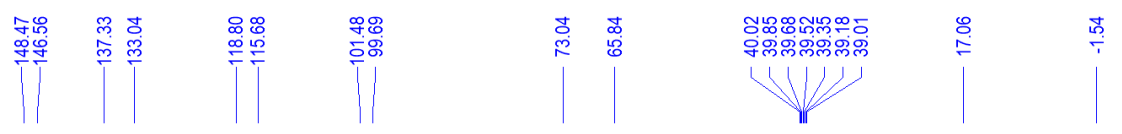<smiles>C[Si](C)(C)CCOCn1ccc2c(Cl)c(C#N)cnc21</smiles>

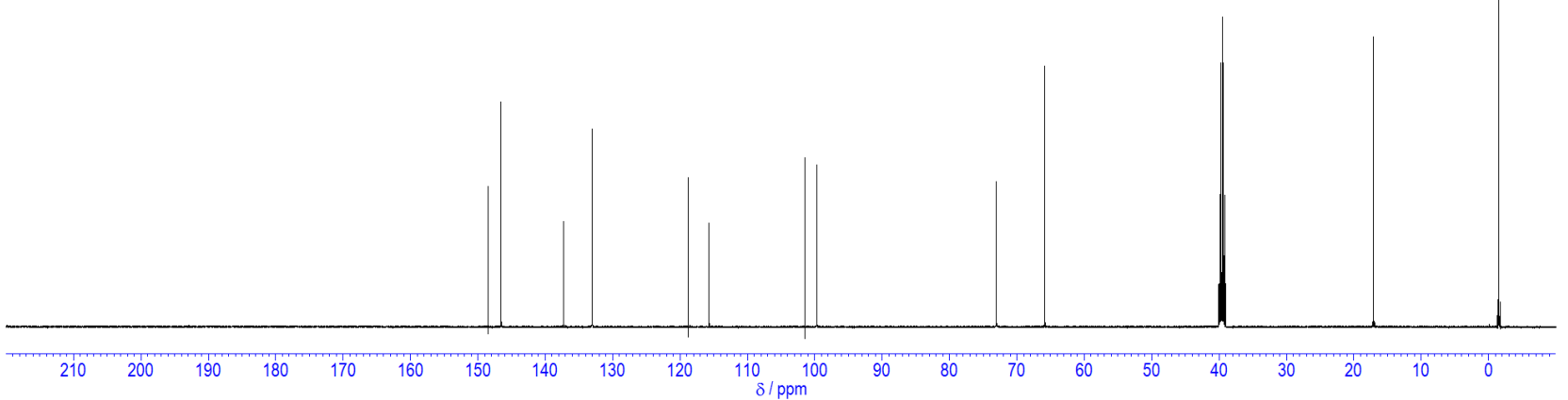




\section{Compound $(3 R, 4 R)-5$}

${ }^{1} \mathrm{H}$ NMR (DMSO-d6, $500 \mathrm{MHz}$ )
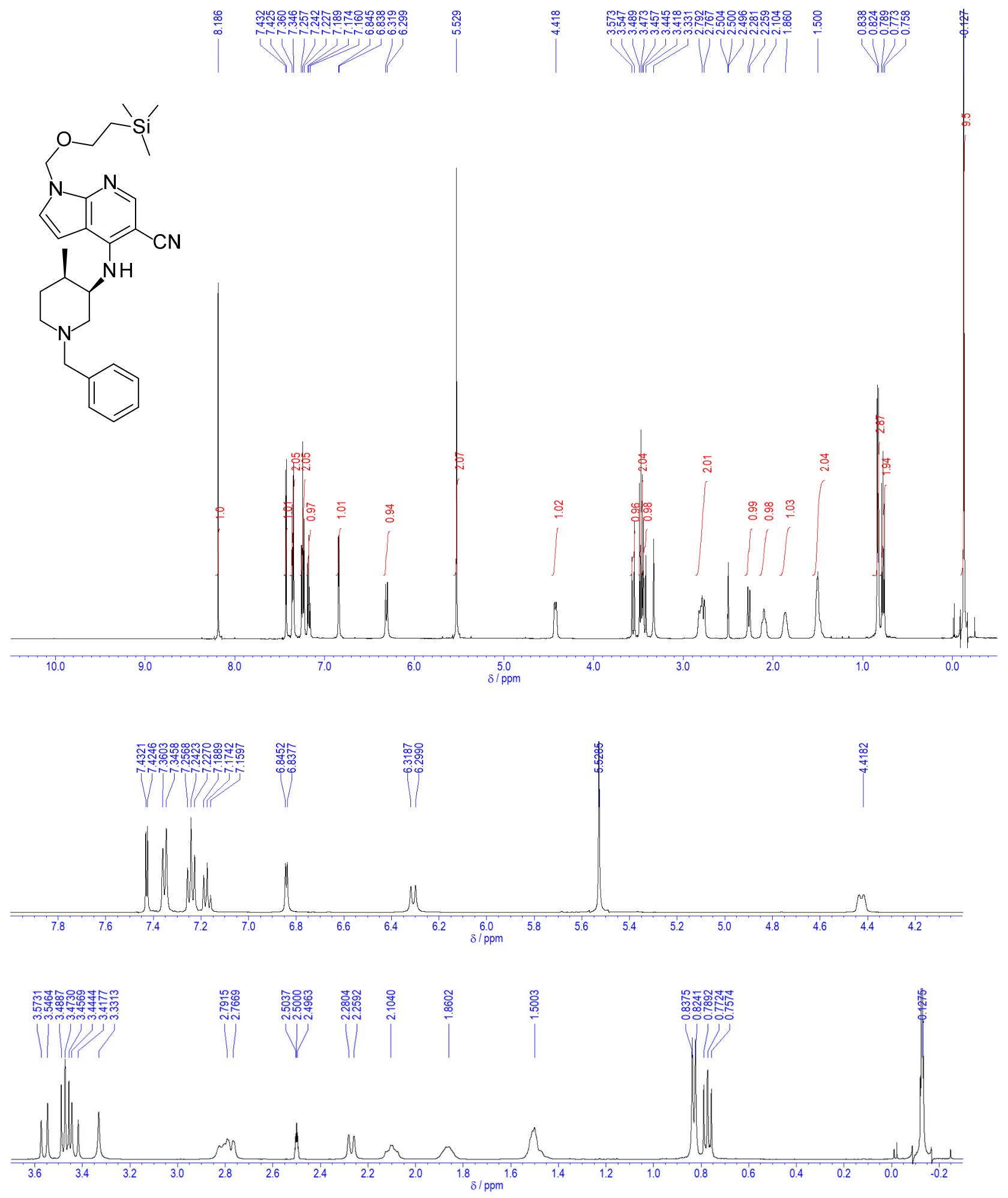


\section{Compound $(3 R, 4 R)-5$}

${ }^{13} \mathrm{C}$ NMR (DMSO-d6, $\left.125 \mathrm{MHz}\right)$

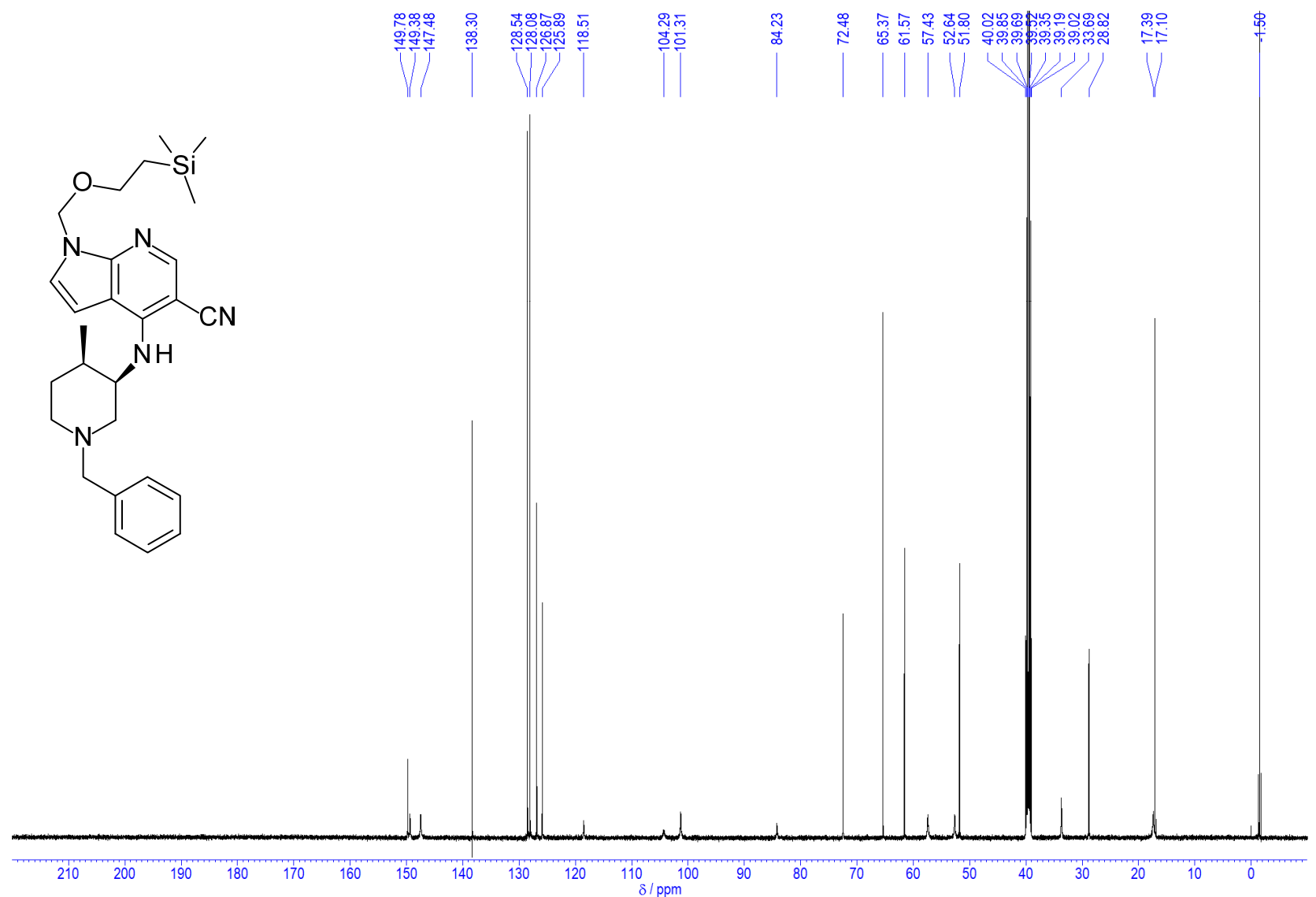


Compound $(3 R, 4 R)-9$

${ }^{1} \mathrm{H}$ NMR (DMSO-d6, $500 \mathrm{MHz}$ )
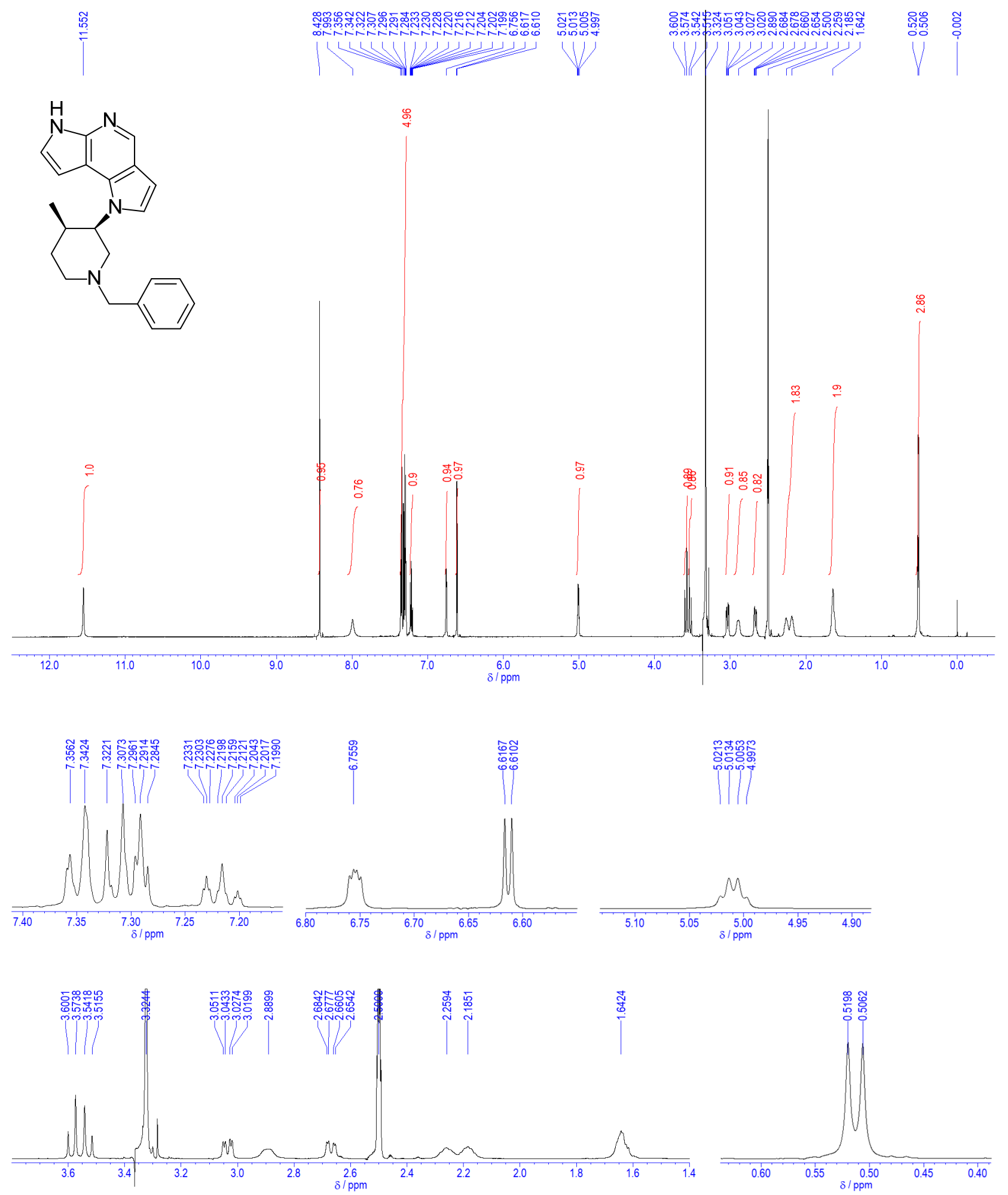
Compound $(3 R, 4 R)-9$

${ }^{13} \mathrm{C}$ NMR $\left(\mathrm{CDCl}_{3}, 125 \mathrm{MHz}\right)$
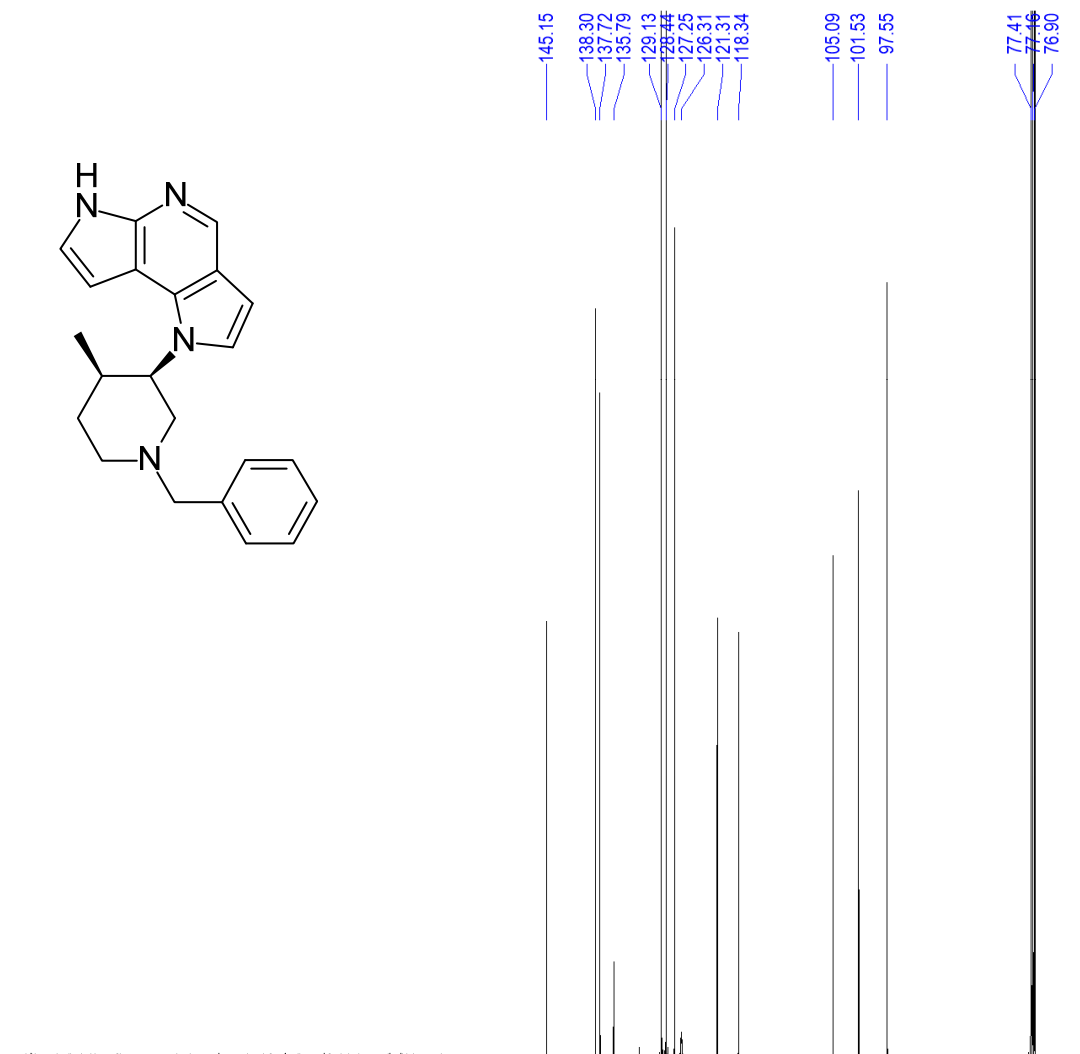

品 5000

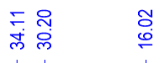

| $\mid$

||

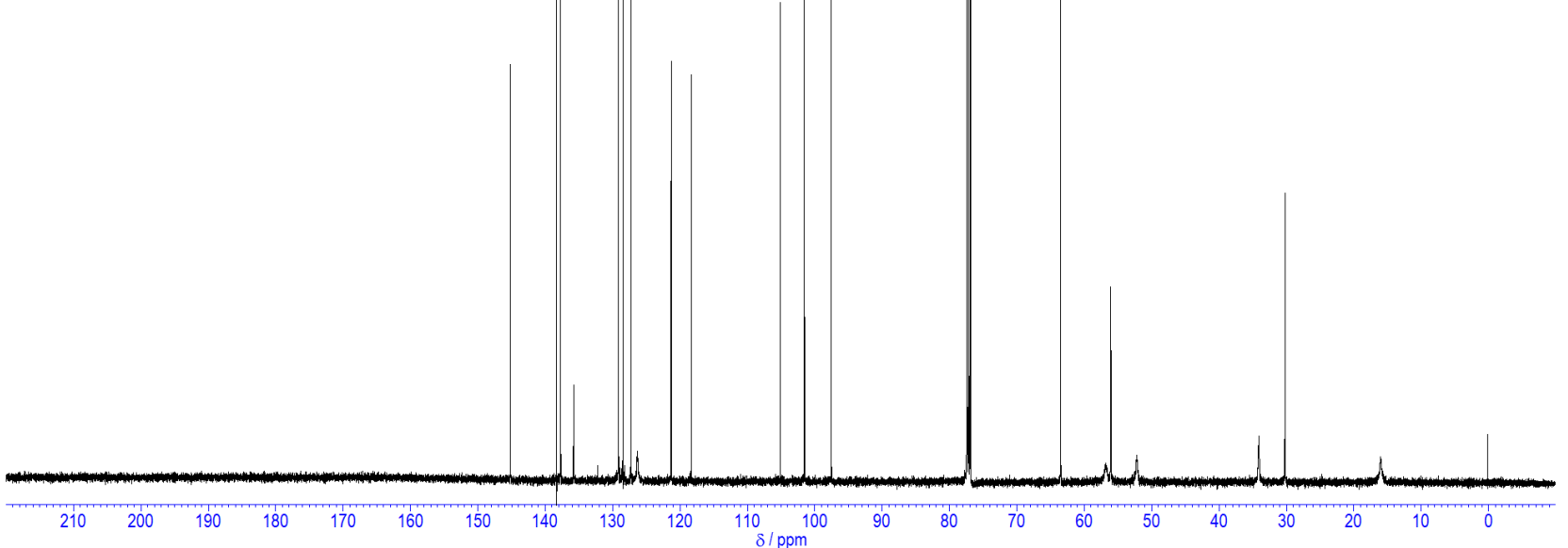




\section{Compound $(3 R, 4 R)-\mathbf{1 0}$}

${ }^{1} \mathrm{H}$ NMR (DMSO-d6, $500 \mathrm{MHz}$ )
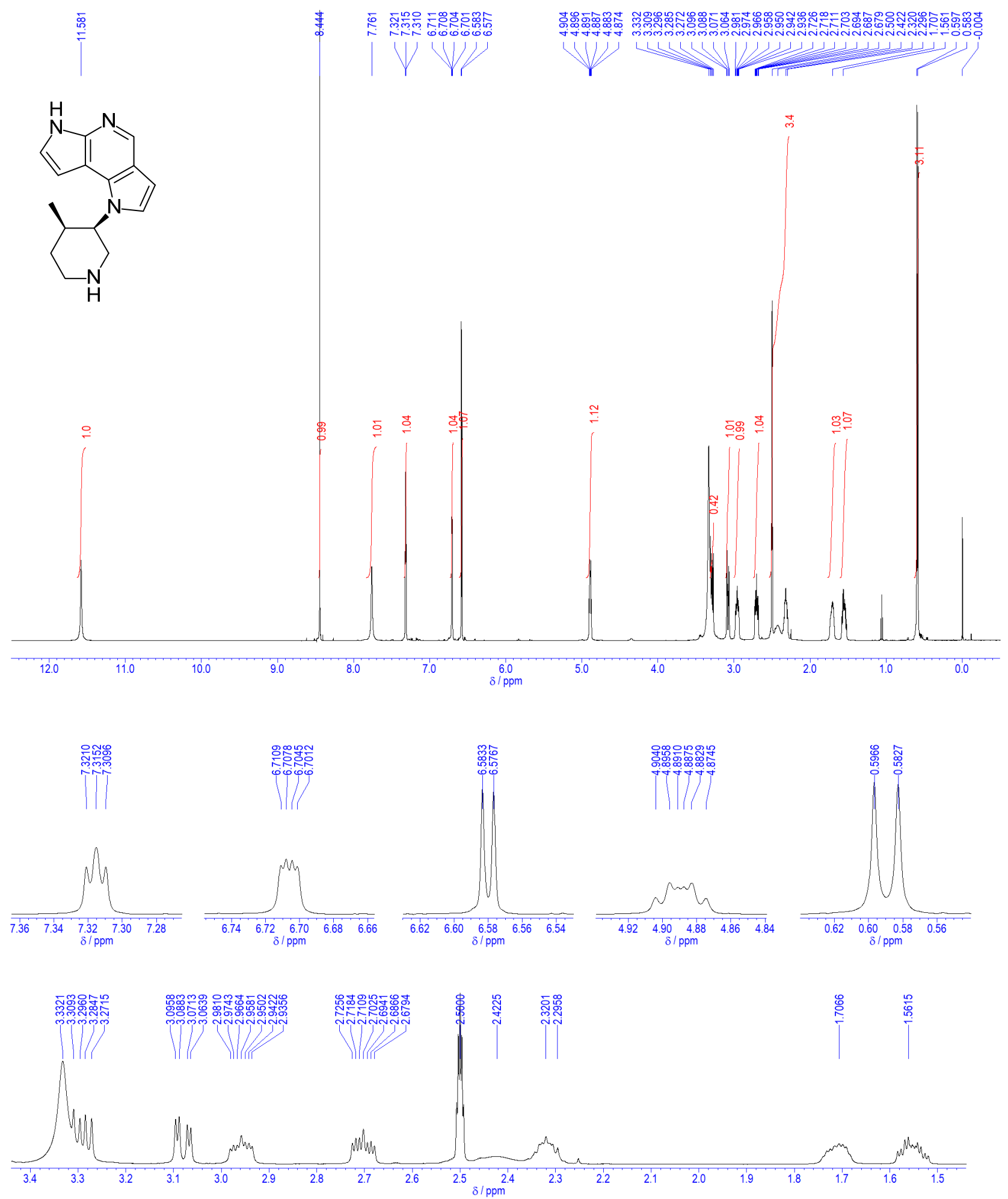
Compound $(3 R, 4 R)-\mathbf{1 0}$

${ }^{13} \mathrm{C}$ NMR (DMSO- $d_{6}, 125 \mathrm{MHz}$ )

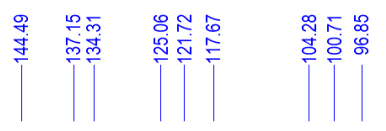

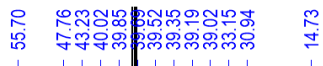<smiles>CC1CCNCC1n1ccc2cnc3[nH]ccc3c21</smiles> 


\section{Compound 1}

${ }^{1} \mathrm{H}$ NMR (DMSO- $d 6,500 \mathrm{MHz}, 27{ }^{\circ} \mathrm{C}, 6 / 4$ mixture of rotamers)
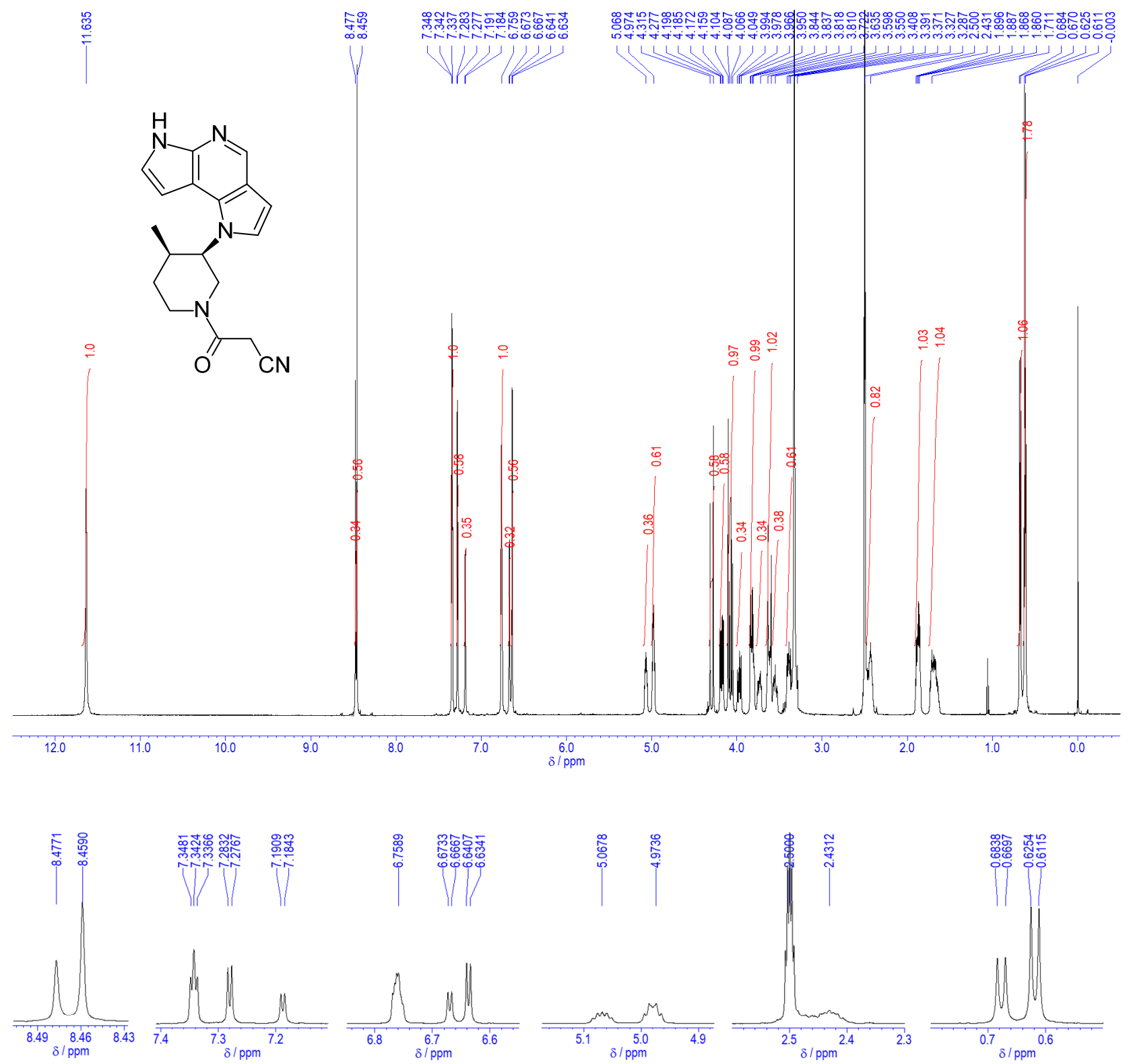

|ll|

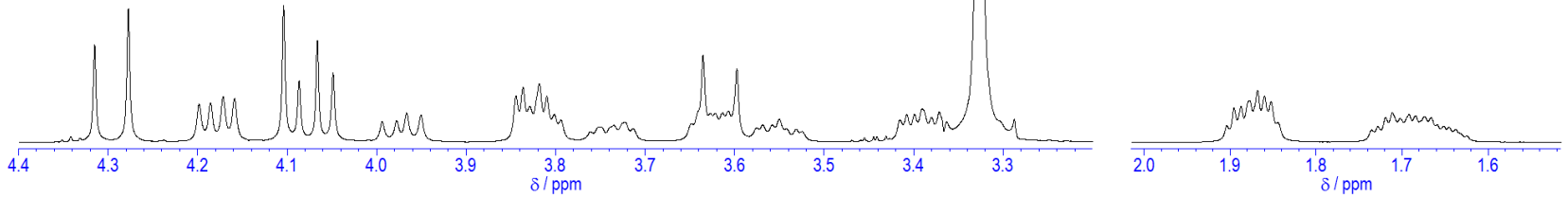




\section{Compound 1}

${ }^{1} \mathrm{H}$ NMR (DMSO- $d 6,500 \mathrm{MHz}, 121{ }^{\circ} \mathrm{C}$ )

$\stackrel{\infty}{=}$

年年

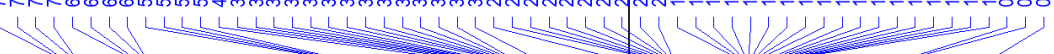<smiles>CC1CCN(C(=O)CC#N)CC1n1ccc2cnc3[nH]ccc3c21</smiles>
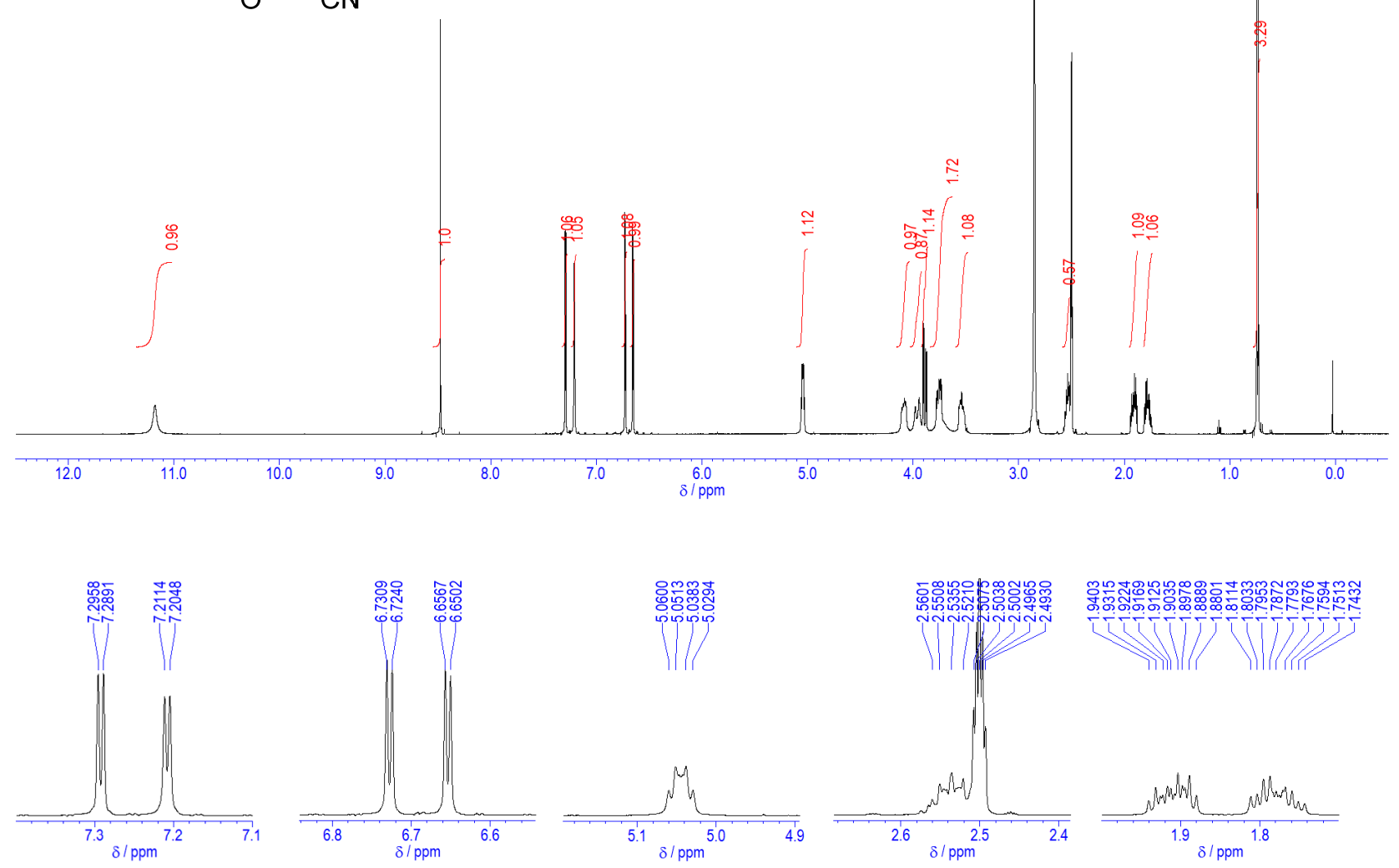

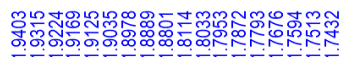

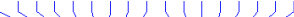

จ
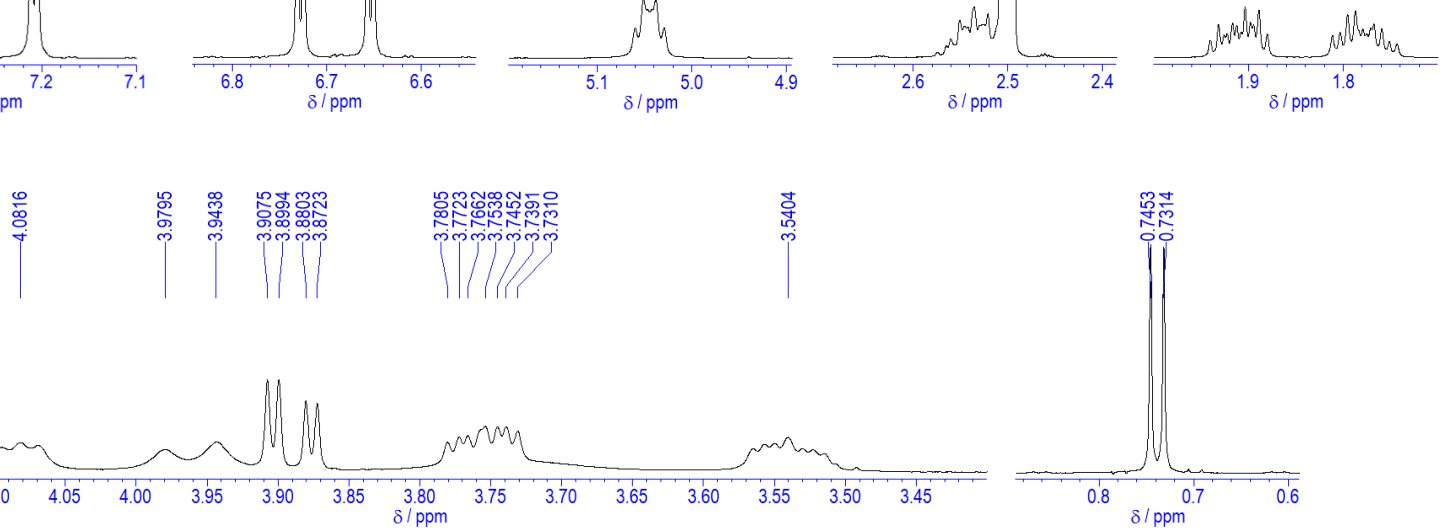
Compound 1

${ }^{13} \mathrm{C}$ NMR (DMSO- $d_{6}, 125 \mathrm{MHz}, 27{ }^{\circ} \mathrm{C}, 6 / 4$ mixture of rotamers)

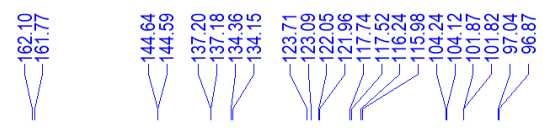

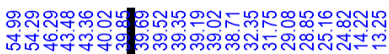
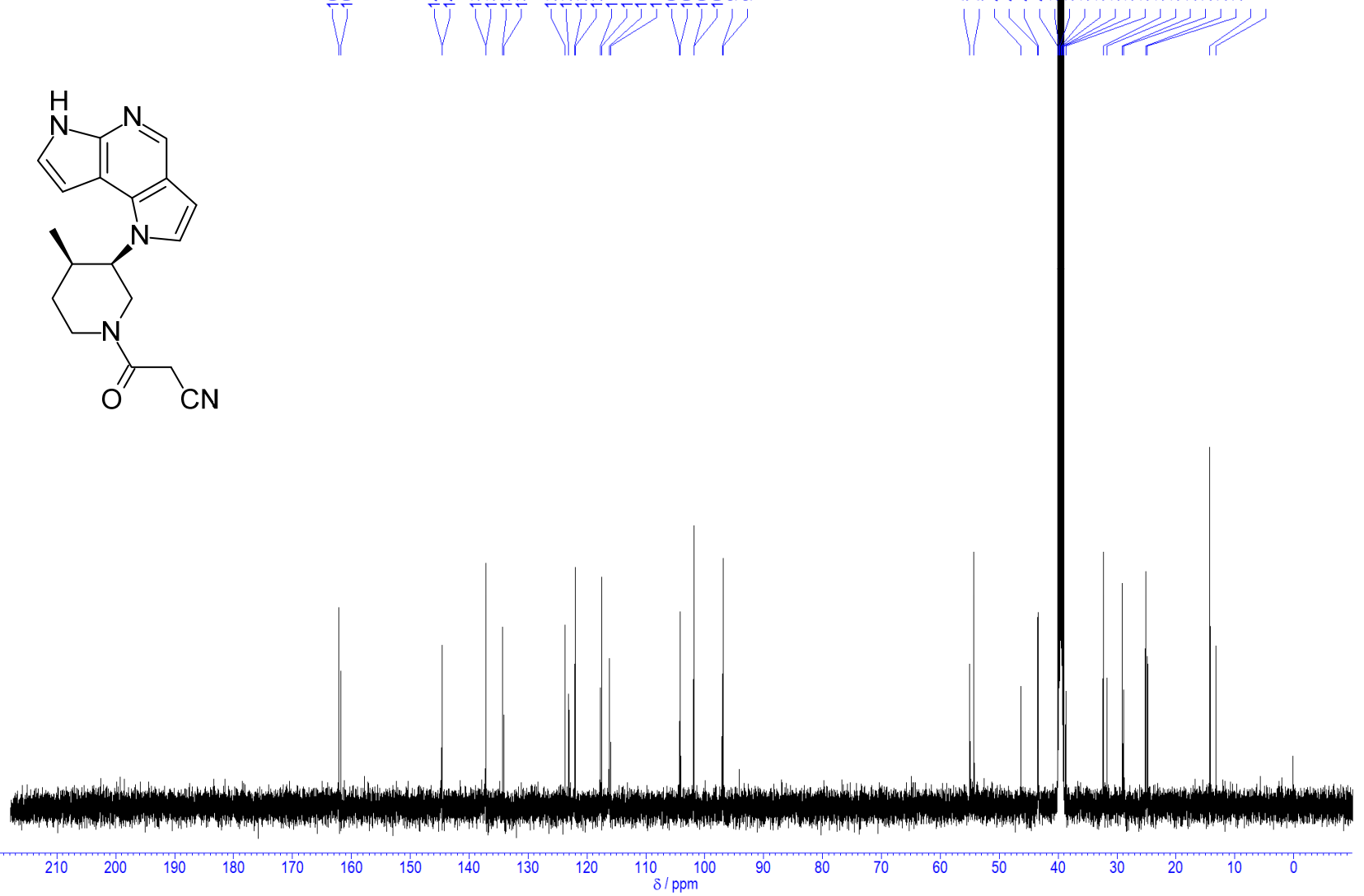